\title{
Reduced White Matter Integrity Is Related to Cognitive Instability
}

\author{
Anders. M. Fjell, Lars. T. Westlye, Inge K. Amlien, and Kristine B. Walhovd \\ Center for the Study of Human Cognition, Department of Psychology, University of Oslo, 0317 Oslo, Norway
}

Increased performance variability has been demonstrated in several groups and conditions, including aging and cognitive decline. Structural brain characteristics underlying this phenomenon have so far been elusive. However, there is reason to expect that disconnectivity in associative pathways, whether caused by immature or degraded white matter (WM) tracts, will increase performance variability by neural noise. The aim of this study was to test whether the quality of WM, measured by diffusion tensor imaging, is related to performance variability in healthy adults. Intraindividual standard deviation of the reaction time (sdRT) across trials and median reaction time (mRT) from 270 participants were obtained from a speeded continuous performance task (Eriksen flanker task) with two conditions (congruent, incongruent). Tract-based spatial statistics was used to test the relationship with diffusion characteristics [fractional anisotropy (FA), mean diffusion (MD), radial diffusion (RD), axial diffusion (AD)]. Robust relationships between sdRT and all diffusion measures were found in most WM areas, independently of mRT, age, and sex. The effects were anatomically more widespread in the congruent than the incongruent condition, covering almost $50 \%$ of the voxels for RD and MD, and $>25 \%$ of the voxels for FA and AD. Partial betas were in the range $0.45-0.55$, and the strength of the relationships increased significantly with age. For mRT, the effects were smaller and unstable across condition. We concluded that performance variability is a likely consequence of individual differences in WM integrity, and that it is a promising behavioral correlate of individual differences in WM microstructure.

\section{Introduction}

The significance of white matter (WM) integrity for cognitive function is rooted in the spatial distribution of cognitive processes in the brain, involving a complex interplay between multiple areas (Gläscher et al., 2010). Lower fiber integrity may cause less stable flow of electrical currents in dendrites and axons, resulting in neural noise, disruptions of the conduction of action potentials, and possibly increased intraindividual variability (IIV) in task performance (MacDonald et al., 2009). The relevance of IIV as a measure of CNS instability has been demonstrated in several groups and conditions, e.g., healthy aging, cognitive decline, ADHD, Parkinson's disease, and frontotemporal dementia (Hultsch and MacDonald, 2004). It is suggested that rapid changes in task performance from one moment to the next reflect mechanisms such as fluctuations in the connectivity of neuronal pathways (Kelly et al., 2008) and the efficacy of neurotransmitter systems (Bäckman et al., 2006). MacDonald and colleagues $(2006,2009)$ further proposed that disconnectivity in associative pathways, whether caused by immature or degraded white matter tracts, can increase variability by neural noise. This would lead to less distinct cortical representations, poorer cogni-

Received Sept. 16, 2011; revised 0ct. 25, 2011; accepted 0ct. 31, 2011.

Author contributions: A.M.F., L.T.W., and K.B.W. designed research; A.M.F., L.T.W., I.K.A., and K.B.W. performed research; A.M.F., L.T.W., and I.K.A. analyzed data; A.M.F. and K.B.W. wrote the paper.

This work was supported by grants from the Norwegian Research Council (204966 to L.T.W., 177404/W50 to K.B.W., and 175066/D15 to A.M.F.) and the University of 0slo (to K.B.W. and A.M.F).

Correspondence should be addressed to Anders M. Fjell, Department of Psychology, Pb. 1094 Blindern, 0317 0slo, Norway. E-mail: andersmf@psykologi.uio.no.

DOI:10.1523/JNEUROSCI.4735-11.2011

Copyright $\odot 2011$ the authors $\quad 0270-6474 / 11 / 3118060-13 \$ 15.00 / 0$ tive performance, and increased performance variability, suggesting that lower WM integrity may be associated with IIV. More than a dozen studies have reported relationships between mental processing speed and diffusion tensor imaging (DTI) (O'Sullivan et al., 2001; Madden et al., 2004, 2009; Schulte et al., 2005; Deary et al., 2006; Grieve et al., 2007; Bucur et al., 2008; Correia et al., 2008; Turken et al., 2008; Kennedy and Raz, 2009; Vernooij et al., 2009), but data are lacking for IIV. Response variability has been related to WM volume (Anstey et al., 2007; Walhovd and Fjell, 2007; Ullén et al., 2008) and hyperintensities (Bunce et al., 2007). However, it needs to be tested whether a relationship between IIV and WM microstructure exists independently of mean reaction time (RT), whether it is a stable trait replicable across tasks, and whether it is modulated by a common influence of age.

Thus, our aim was to test the relationship between IIV and WM integrity. The main hypothesis was that IIV would be negatively associated with diffusion anisotropy [fractional anisotropy (FA)] and positively with amount of diffusion [mean diffusion $(M D)$, radial diffusion (RD), axial diffusion $(A D)]$ in widespread areas. Second, we wanted to test to what extent IIV-WM relationships were replicable across conditions with different cognitive load. Since frontal lesions increase IIV (Stuss et al., 2003) and attention failures may be an important source of increased IIV (West et al., 2002), increased cognitive load may contribute to a more anterior distribution of effects. The complex condition also involves inhibitory processing, which further increases the demands for involvement of prefrontal brain structures. Finally, we tested whether the IIV-WM relationship changed with increasing age. As WM microstructure is known to deteriorate substan- 
Table 1. Sample characteristics

\begin{tabular}{lcc}
\hline & Mean (SD)/frequency & Correlation with age \\
\hline$N$ & 270 & \\
Age (years) & $48.6(20-83)$ & \\
Sex & 154 females/116 males & 0.03 \\
Education $^{1}$ & $15.6(2.7)$ & -0.01 \\
MMSE $^{2}$ & $29.2(0.8)$ & $-0.25^{*}$ \\
Full-scale IQ & $114.3(9.0)$ & 0.09
\end{tabular}

${ }^{*} p<0.05$.

${ }^{1}$ Available for 268 participants only.

${ }^{2}$ Available for participants $>30$ years of age only

tially through the adult lifespan (Westlye et al., 2010), we hypothesized that the relationship with IIV would increase in strength with age.

\section{Materials and Methods}

Sample. The sample was drawn from the first wave of a longitudinal research project at the Center for the Study of Human Cognition at the University of Oslo called Cognition and Plasticity through the Life-Span. The study was approved by the Regional Ethical Committee of Southern Norway. The participants were recruited through newspaper ads and among students and employees of the University of Oslo. Further details regarding recruitment and enrolment were given previously (Fjell et al., 2008; Westlye et al., 2009). Written informed consent was obtained from all participants. Two hundred seventy healthy participants $(57.0 \%$ females), aged 20-83 years (mean, 48.6 years; SD, 16.9 years) were included. There was no correlation between sex and age (Pearson's $r=$ $0.03, p>0.60$, with females coded as 0 and males as 1 ). All subjects were right-handed native Norwegian speakers. Participants were screened using a standardized health interview on telephone before inclusion in the study. History of self-reported neurological or psychiatric conditions thought to affect normal cerebral functioning, including clinically significant stroke, traumatic brain injury, untreated hypertension, diabetes, and use of psychoactive drugs within the last two years, were exclusion criteria. Further, participants reporting worries concerning their cognitive status, including memory function, were excluded. All participants scored $<16$ on Beck Depression Inventory (Beck and Steer, 1987). Participants $>30$ years of age were also tested with Mini Mental State Examination (MMSE) (Folstein et al., 1975; Bravo and Hébert, 1997) and all scored $\geq 26$. General cognitive abilities were assessed by Wechsler Abbreviated Scale of Intelligence (WASI) (Wechsler, 1999). All subjects scored within normal IQ range (82-145). We believe that the participants were healthy, but it is difficult to ascertain that none would be in the very early stage of age-related neurodegenerative condition, as the progression of such diseases as Alzheimer's disease is very slow without clinical symptoms in the initial phases. Ideally, the participants should be followed for several years to exclude the possibility of incipient disease.

All participants' MR scans were examined by a specialist in neuroradiology and all included participants had to be deemed free of significant anomalies, including signs of vascular insults. A total of 285 participants were enrolled in the study. After the initial health screening, 15 participants were excluded due to missing MRI data or motion artifacts, lack of valid reaction time data, age (one participant exceeded 90 years, which created a gap of missing data points on the continuous age scale), or after radiological evaluation (two participants), yielding a final sample of 270 subjects. Sample characteristics are shown in Table 1.

Experimental task. We administered a modified version of the Eriksen flanker task (Eriksen and Eriksen, 1974), described in detail previously (Westlye et al., 2009), similar to the task used by Debener et al. (2005). An illustration of the task is shown in Figure 1. The stimuli were horizontal arrows (length, $1^{\circ}$ ), pointing either to the right or the left, and displayed in a vertical stack $2.5^{\circ}$ high. Subjects were instructed to respond as accurately and quickly as possible by button press indicating which direction the middle arrow was pointing. Each trial consisted of the following stimuli. First, a fixation cross was presented for a random interval ranging between 1200 and $1800 \mathrm{~ms}$. Then the four flanker arrows were pre- sented for $80 \mathrm{~ms}$ before the target arrow was presented for $30 \mathrm{~ms}$ along with the flanker arrows. The flanker arrows were presented before the target to increase prepotent responding and to make the task more difficult. At presentation of the target, the task was to push one button if the target was pointing to the left and another button if the target was pointing to the right. Based on the mean RT for the first 20 consecutive trials, an individually adjusted RT criterion ( $10 \%$ above mean RT of the 20 initial trials) was set. After every subsequent third trial with either RT exceeding this criterion or with response omission, a message occurred on screen for $1 \mathrm{~s}$ instructing the participant to respond faster. This was also implemented to increase the demand to respond swiftly and thus increase task difficulty. Before the experiment, a training session of 20 trials was administered to familiarize the participant with the task.

On average, 7.7 instructions to respond faster were given during the complete experiment ( $\mathrm{SD}=13.7$ ). As one would expect, number of instructions correlated with standard deviation of the RT (sdRT; $r=0.63$ and 0.46 in the congruent and incongruent conditions, respectively). That is, participants with a more variable response style would more often exceed the individually adjusted RT threshold. The number of instructions did not correlate with intellectual ability as quantified by full-scale IQ from WASI $(r=-0.00, p=0.96)$, education $(r=-0.01$, $p=0.92)$, or MMSE score $(r=-0.01, p=0.86)$, or with age when median RT (mRT) was partialled out $(r=0.00, p=0.98)$. The rational for using this procedure was to increase the participants' motivation for rapid responses and to enhance their attentional investments in the task. We expected that this would lead to somewhat lower sdRT, reduce variability due to random attentional drifts, and leave us with a measure of variability more closely related to task-focused CNS function. Thus, sdRT in this task may be more related to the ability to respond in a constant and speedy manner, rather than naturally occurring trial-totrial variability.

Responses were obtained on a PST Serial Response Box and the experimental procedures and responses were collected using E-prime software (Psychological Software Tools). There were two experimental task conditions: congruent and incongruent. In the congruent condition, all arrows pointed in the same direction. In the incongruent condition, the middle arrow pointed in the direction opposite of that of the flanker arrows. The task included 416 trials with a short break halfway. The probability of an incongruent trial was 50\% in a randomized fashion.

This and similar tasks have been shown to produce the well described congruency effect with higher RT and lower accuracy in the incongruent compared with the congruent condition (Eriksen and Eriksen, 1974). The congruency effect is probably reflecting response conflict induced in the incongruent condition (Botvinick et al., 2001). Responses to incongruent stimuli reflect more complex cognitive processing, including inhibitory processes and generally more top-down and controlled attention than the much simpler responses to the congruent stimuli. Differences in conscious attention and response strategies will likely also influence the results. We report results both from the cognitively simple congruent condition and from the more complex incongruent condition to enable comparison of the relationship between IIV and WM microstructure across conditions of different complexity and different cognitive demands.

Congruent and incongruent trials (208 each) were administered. For statistical analyses, we excluded the first 10 trials and the 10 trials with the fastest and slowest RTs, as it is difficult to decide whether extreme responses represent variations of the real cognitive processes under study, or whether they result from random noise due to factors such as the participant missing the button, having a single lapse of attention during the course of a long speeded task, etc. Thus, a simple way of reducing the possibility that such noise contaminate the data, without biasing the results in either direction, is to exclude the extreme ends of the RT distribution for all participants. Further, trials after which the participants made an error were excluded due to the possibility of posterror slowing affecting the results. For the resulting trials, $\mathrm{mRT}$ and sdRT were calculated and used as measures of interest. Median RT was preferred to mean RT since RT generally does not follow strict normal distribution but has a thicker tail of slow compared with fast values. However, in the present dataset, the correlation between the median and the mean RT was 0.995 
for the congruent and 0.989 for the incongruent conditions, indicating that the practical implications of choosing the one over the other are negligible for the present data. The correlation between mean RT across subjects per trial and trial number was $r=0.04(p=0.51)$, indicating that no clear linear drift in RT existed throughout the course of the task session. This is probably partly due to the training session administered before the experiment, the feedback presented on screen in response to slow RTs or response omissions (see above), and task-related factors.

MR acquisition. Imaging data were collected using a 12-channel head coil on a $1.5 \mathrm{~T}$ Siemens Avanto scanner (Siemens Medical Solutions) at Rikshospitalet University Hospital, Oslo. For diffusion-weighted imaging, a single-shot twice-refocused spin echo EPI pulse sequence with 30 diffusion-sensitized gradient directions was used (TR, $8200 \mathrm{~ms}$; TE, $82 \mathrm{~ms} ; b$ value, $700 \mathrm{~s} / \mathrm{mm}^{2}$; voxel size, $2.0 \times 2.0 \times 2.0$ $\mathrm{mm})$. This sequence was optimized to minimize eddy current-induced image distortions (Reese et al., 2003). The sequence was repeated in two successive runs with $10 b=0$ in addition to 30 diffusion-weighted images collected per acquisition. Each volume consisted of 64 axial slices. Total scanning time was $11 \mathrm{~min}, 21 \mathrm{~s}$.

The two acquisitions were concatenated during postprocessing to increase signal-tonoise-ratio. All datasets were processed and analyzed at the Neuroimaging Analysis Lab, Center for the Study of Human Cognition, University of Oslo, with additional use of computing resources from the Titan High Performance Computing facilities (http:// hpc.uio.no/index.php/Titan) at the University of Oslo.

$M R$ analysis. Image analyses and tensor calculations were done using FSL (http://www.fmrib. ox.ac.uk/fsl/index.html) (Smith et al., 2004; Woolrich et al., 2009). Initially, each DTI volume was affine registered to the T2-weighted $b=0$ volume using FLIRT (Jenkinson and Smith, 2001). This corrected for motion between scans and residual eddy-current distortions present in the diffusion-weighted volumes. To preserve the orientational information after motion correction, we reoriented each volume's B matrix by applying the corresponding transformation matrix from the motion-correction procedure. After removal of nonbrain tissue (Smith, 2002), least-square fits were performed to estimate the FA and eigenvector and eigenvalue maps. We defined $\mathrm{RD}$ as the mean of the second and third eigenvalue $\left(\left(\lambda_{2}+\lambda_{3}\right) / 2\right)$ and MD as the mean of all three eigenvalues. Next, all individuals' FA volumes were skeletonized and transformed into a common space as used in tract-based spatial statistics (TBSS) (Smith et al., 2006, 2007). Briefly, all volumes were nonlinearly warped to the FMRIB58_FA template, which is supplied with FSL, by use of local deformation procedures performed by FNIRT (Andersson et al., 2007a,b), a nonlinear registration toolkit using a $b$-spline representation of the registration warp field (Rueckert et al., 1999).

The common template used in the present study is a highresolution average of $58 \mathrm{FA}$ volumes from healthy male and female subjects aged 20-50 years. It is expected that the amount of warping needed to align each individual FA volume to this template would commensurate with the distance in each participant's age from the age of the subject's used to build the template. All warped FA volumes were visually inspected for accuracy, which is especially pertinent when analyzing life-span datasets with relatively large individual variability in brain size and architecture. We have previously shown that

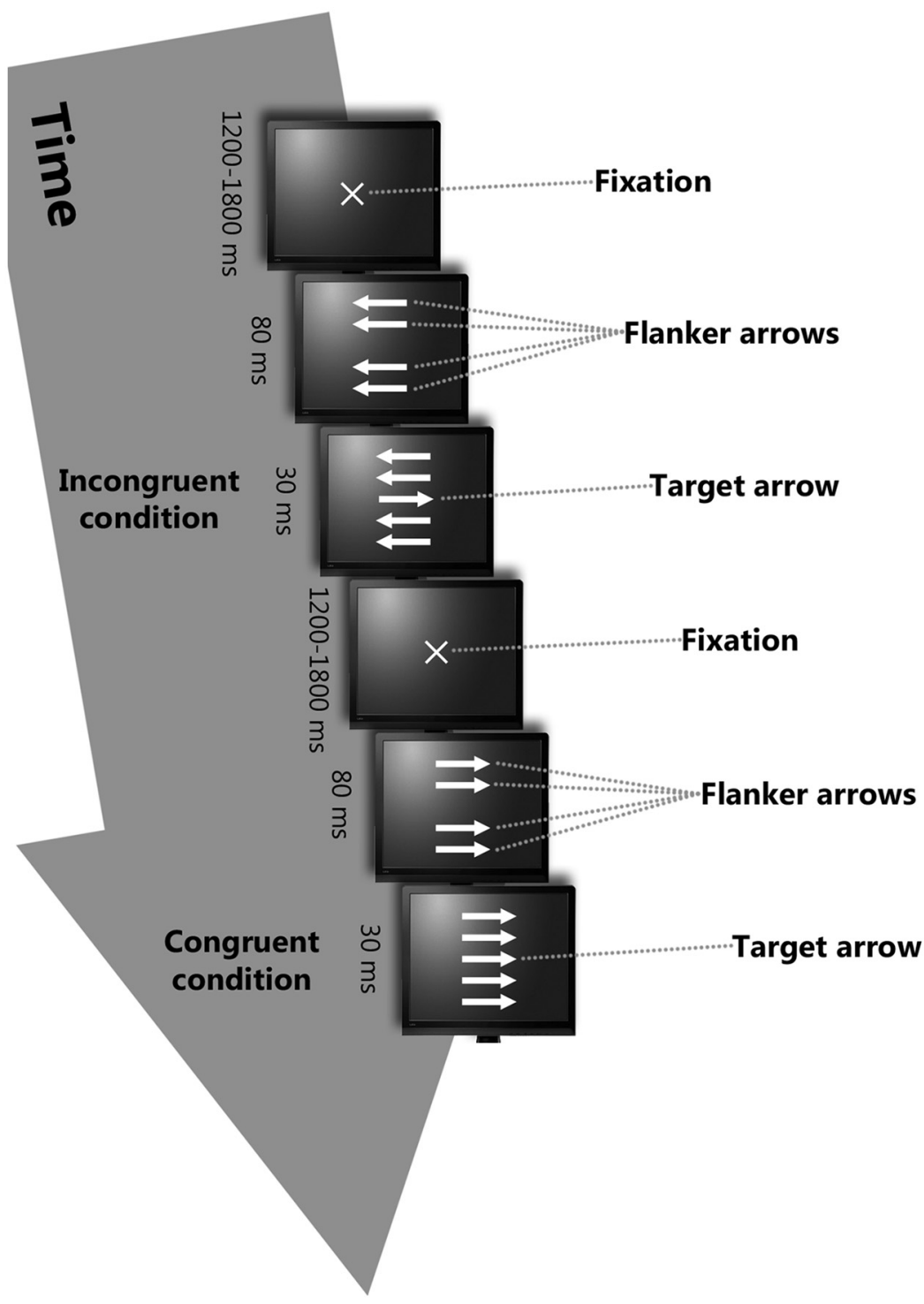

Figure 1. Schematic overview of the Flanker task.

FNIRT performed the native-to-standard warping adequately across age groups (Westlye et al., 2010).

Next, a mean FA volume of all subjects was generated and thinned to create a mean FA skeleton representing the centers of all common tracts. We binarized the mean skeleton at FA threshold $>0.2$ to reduce the likelihood of partial voluming in the borders between tissue classes, yielding a mask of 128,142 WM voxels. Individual FA values were warped onto this mean skeleton mask by searching perpendicular from the skeleton for maximum FA values. Using maximum FA values from the centers of the tracts further minimizes confounding effects due to partial voluming (Smith et al., 2006). The resulting tract invariant skeletons for each participant were fed into voxelwise permutation-based crosssubject statistics to test for age-related changes in WM integrity. Similar warping and analyses were used on $\mathrm{AD}, \mathrm{RD}$, and $\mathrm{MD}$ data, yielding $\mathrm{RD}$ and MD skeletons sampled from voxels with FA $>0.20$. Areas showing evidence of white-matter hyperintensities (WMH) were not excluded in the present study. The skeleton approach and the FA threshold used will likely reduce possible confounds from WMH, yet we cannot exclude the possibility that hyperintensities might have affected the FA values in specific parts of the skeleton for some of the subjects, in particular in periventricular areas in the oldest participants. This limitation should be addressed in future studies by using specific MR sequences for WMH segmentation. 
Statistics. Voxel-based DTI analyses were performed using permutation testing (Nichols and Holmes, 2002), as implemented in randomize, part of FSL. We tested for linear effects of mRT on FA, RD, AD, and MD with general linear models (GLM) while regressing out the effects of sex and age, and we tested for effects of sdRT on the four DTI parameters regressing out sex, age, and $\mathrm{mRT}$. The research community has not established a standard for calculating intraindividual trial-to-trial variability, and several approaches exist, including intraindividual SD and intraindividual coefficient of variation (Lövdén et al., 2007). In the present study, intraindividual SD was used. mRT was used as covariates in all analyses involving sdRT since a relationship between $\mathrm{mRT}$ and sdRT is expected. Not controlling for mRT could yield the trivial result of older participants with higher $\mathrm{mRT}$ also exhibiting higher IIV (for a discussion of these and other issues related to quantification of IIV, see MacDonald et al., 2009). Threshold-free cluster enhancement (Smith and Nichols, 2009) with default settings was used for statistical inference, with 5000 permutations performed for each contrast. Statistical $p$ value maps were thresholded at $p<0.05$, corrected for multiple comparisons across space. For each DTI parameter, values were extracted from significant skeleton voxels and plotted against sdRT and mRT to illustrate the individual data points and to provide a general measure of effect size. As these correlation analyses were restricted to voxels where the various diffusion values were known to be significantly related to IIV, these must not be regarded as part of the hypothesis testing, but only as a convenient way of estimating effect sizes for comparison with other studies. We used standardized partial betas from hierarchical liner regression analyses to illustrate the strength of the relationship between the diffusion parameters and mRT with effects of age and sex regressed out, and between the diffusion parameters and sdRT with effects of age, sex, and $\mathrm{mRT}$ regressed out.

Finally, randomize was run for each diffusion parameter to explicitly test whether the relationship between sdRT and diffusion parameters changed with age. The age $\times$ sdRT interaction term was included in the analyses, with sex, age, mRT, and sdRT used as covariates. Finally, the sample was divided by the median age and the main effects were replicated. This was to illustrate the result of the interaction analysis and to replicate the results in more age-homogenous samples (for a more detailed discussion of this point, see Hofer and Sliwinski, 2001).

\section{Results \\ Description of task performance}

Task performance accuracy in the congruent condition was high, with mean correct responses to congruent trials of 95.4\% ( SD = 7.8), yielding a mean number of correct congruent trials of 198.3 $(S D=16.3)$ of a total of 208. After excluding the first 10 trials and the 10 trials with the fastest and slowest RTs for each participant, and all trials following an error response, the mean number of trials entered into statistical analyses was $163.9(\mathrm{SD}=27.5)$. Mean $\mathrm{mRT}$ was $384 \mathrm{~ms}(\mathrm{SD}=68$, skewness $=0.44$, kurtosis $=$ $1.30)$ and mean sdRT was $58 \mathrm{~ms}(\mathrm{SD}=21$, skewness $=3.04$, kurtosis $=18.54)$.

As expected, mean number of correct responses in incongruent trials was significantly lower $\left(t_{(269)}=22.09, p<10^{-61}\right)$, with a mean of $75.8 \%(S D=16.71)$, a significantly higher $\mathrm{mRT}$ of $473.8 \mathrm{~ms}(\mathrm{SD}=77.2$, skewness $=-0.68$, kurtosis $=3.46, z=$ $\left.-14.18, p<10^{-44}\right)$, and lower sdRT of $51 \mathrm{~ms}$ (SD $=25$, skewness $=3.46$, kurtosis $\left.=20.9, z=-9.56 . \mathrm{p} \ll 10^{-20}\right)$. This shows that the experimental paradigm yields the expected effects. Spearman's rho for mRT across conditions was 0.94 , and 0.80 for sdRT. The high coefficients show that both speed of processing and IIV can be generalized across different conditions. Still, since the correlation for sdRT was far from perfect, all subsequent analyses were run for both conditions.

\section{Relationship with age}

Neither mRT (congruent condition Mann-Whitney $U$ test: $z=$ -0.66 , n.s.; incongruent condition Mann-Whitney $U$ test: $z=$ -0.76, n.s.) nor sdRT (congruent condition Mann-Whitney $U$ test: $z=-0.88$, n.s.; incongruent condition Mann-Whitney $U$ test: $z=-0.66$, n.s.) differed between females and males. Still, sex was used as covariate in all subsequent analyses. Both mRT and sdRT correlated with age (congruent condition mRT: $r=0.66$, $p<10^{-34}$; incongruent condition mRT: $r=0.58, p<10^{-24}$; congruent condition sdRT: $r=0.35, p<10^{-8}$; incongruent condition sdRT: $r=0.24, p<10^{-4}$ ). sdRT and age did not correlate significantly in the congruent condition when mRT was controlled for $(\rho=0.06, p<0.31)$, while a significant correlation was seen in the incongruent condition $(\rho=0.22, p<0.001)$. Still, age was used as covariate together with sex for all analyses involving sdRT.

\section{Relationships between mRT and sdRT from the congruent condition and DTI parameters}

Figure 2 shows the relationships between sdRT from the congruent condition and the different DTI parameters as color-coded overlays in the horizontal plane; scatterplots are shown in Figure 3. The results for mRT are shown in Figure 4. Effects sizes and the spatial extensions of the overall effects are shown in Table 2.

Higher sdRT was significantly associated with all DTI parameters in widespread areas, independently of age, sex, and mRT, covering from $26 \%$ (AD) to almost $50 \%$ (MD) of the skeleton voxels. The relationships with FA were negative, indicating that higher sdRT was associated with lower FA, while the other relationships were positive, indicating that higher levels of sdRT were related to higher degree of diffusion in all directions. No reverse relationships were observed. The areas of effects included both anterior and posterior sections and both dorsal and ventral areas. The effects were seen throughout all major WM tracts. Some regional variability across diffusion parameters was observed, e.g., that the relationships between sdRT and $\mathrm{AD}$ were generally weaker in occipital and posterior-most sections of the WM than what was seen for RD and MD. To directly compare the distribution of effects along the posterior-to-anterior axis across conditions, demeaned (within condition) $t$ scores were plotted as a function of MNI $y$-coordinates; the results are shown in Figure 5. As can be seen, there was no clear tendency for larger effects in anterior versus posterior parts of the WM or vice versa. For MD, the effects of sdRT on diffusion seemed smaller in the middle part of the brain compared with more anterior and posterior areas, while this tendency was much weaker for FA and AD, and not present for RD. No formal statistical test was performed on the distribution of $t$ values along the $y$-axis.

$\mathrm{mRT}$ was related to higher $\mathrm{AD}$ and $\mathrm{MD}$ in the congruent condition, independent of age and sex, but not to FA or RD. mRT was significantly related to $\mathrm{AD}$ in the body and genu of the corpus callosum, as well as a portion of the left anterior thalamic radiation and forceps minor. The relationships with MD were found in the genu and body of corpus callosum. The effects were highly localized, and comprised only $1.9 \%(\mathrm{AD})$ and $0.6 \%$ of the skeleton voxels.

\section{Age interactions}

To test whether the relationship between sdRT from the congruent condition and diffusion parameters changed with age, voxelwise GLMs with an age $\times$ sdRT interaction term included as an additional independent variable were run. The results are shown in Figure 6. Age interactions were seen in large parts of the WM, 


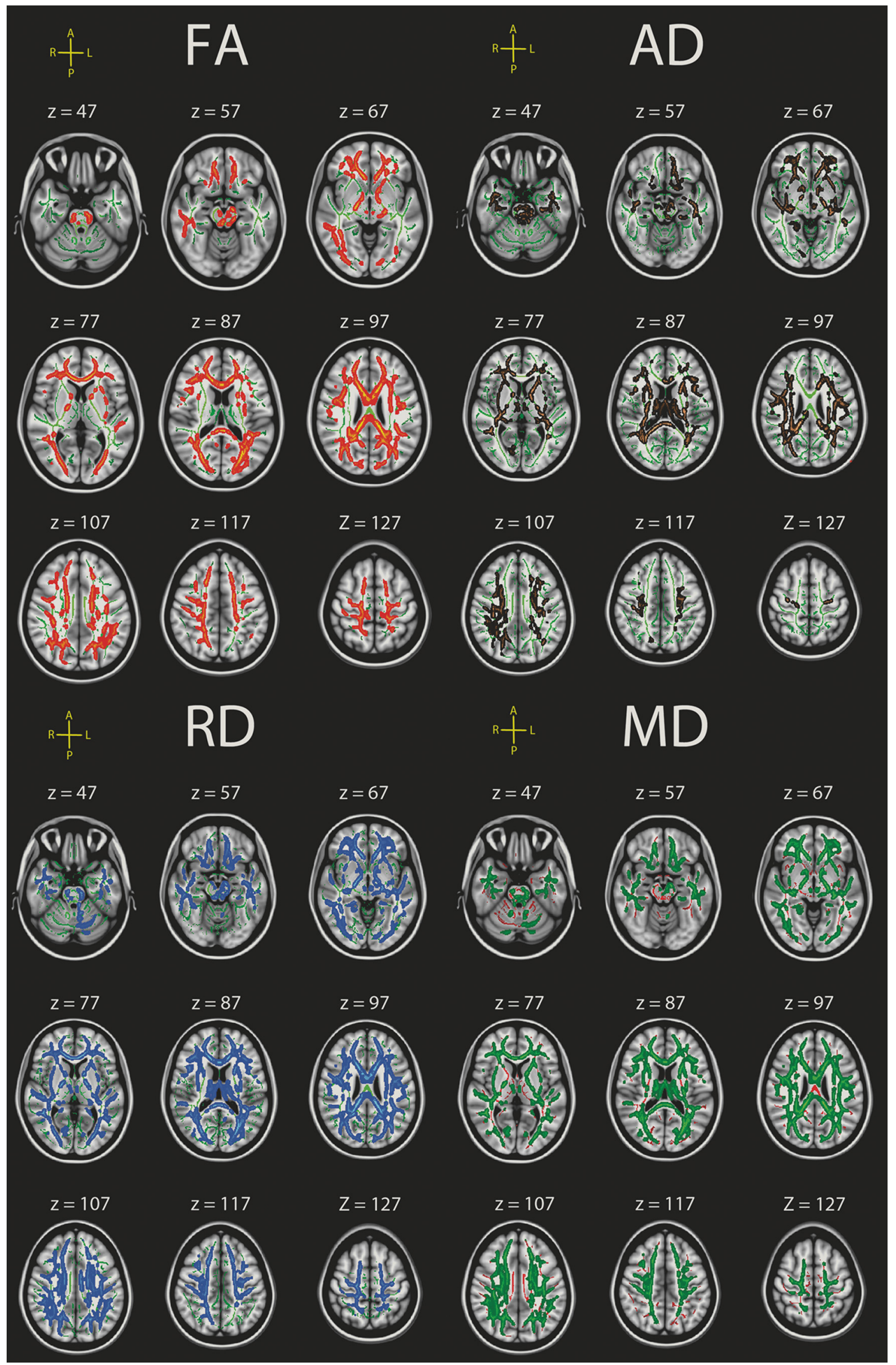

Figure 2. Effects of $s d R T$ in the congruent condition on diffusion characteristics. Effects of IIV on diffusion characteristics, corrected for age, sex and mRT. The effects are corrected for multiple comparisons across space by threshold-free cluster enhancement at $p<0.05$. The results are smoothed to ease visualization of effects, and displayed on top of the white matter skeleton (red on green skeleton, FA; copper on green skeleton, AD; blue on green skeleton, $\mathrm{RD}$; green on red skeleton, $\mathrm{MD}$ ). FA was negatively correlated with sdRT; the other diffusion measures were positively related. 


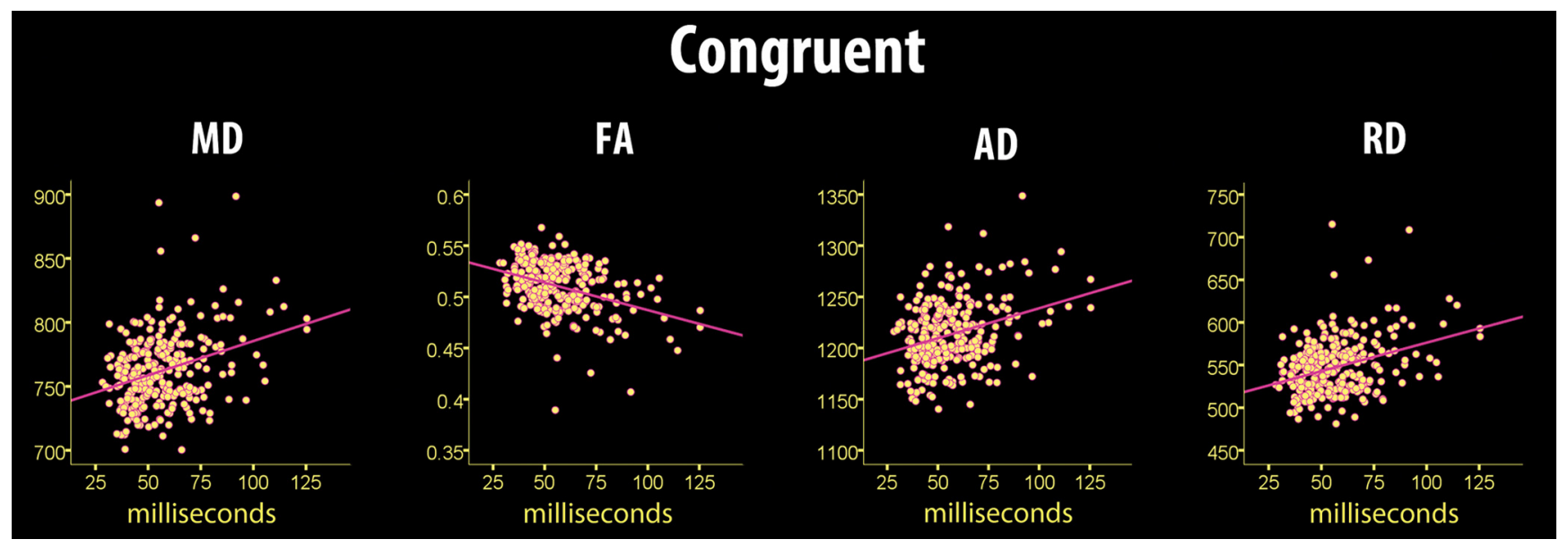

Figure 3. Scatterplots of sdRT in the congruent condition and diffusion characteristics. The scatterplots are used to illustrate the raw data, and thus age, sex, and $\mathrm{mRT}$ are not used as covariates, in contrast to the other statistical analyses. Four outliers were excluded from the analyses (sdRT $\pm 3 S D$ from the mean). The diffusion characteristics represent mean values across all voxels identified as statistically significant in the voxelwise skeleton analyses (fully corrected for multiple comparisons across space).

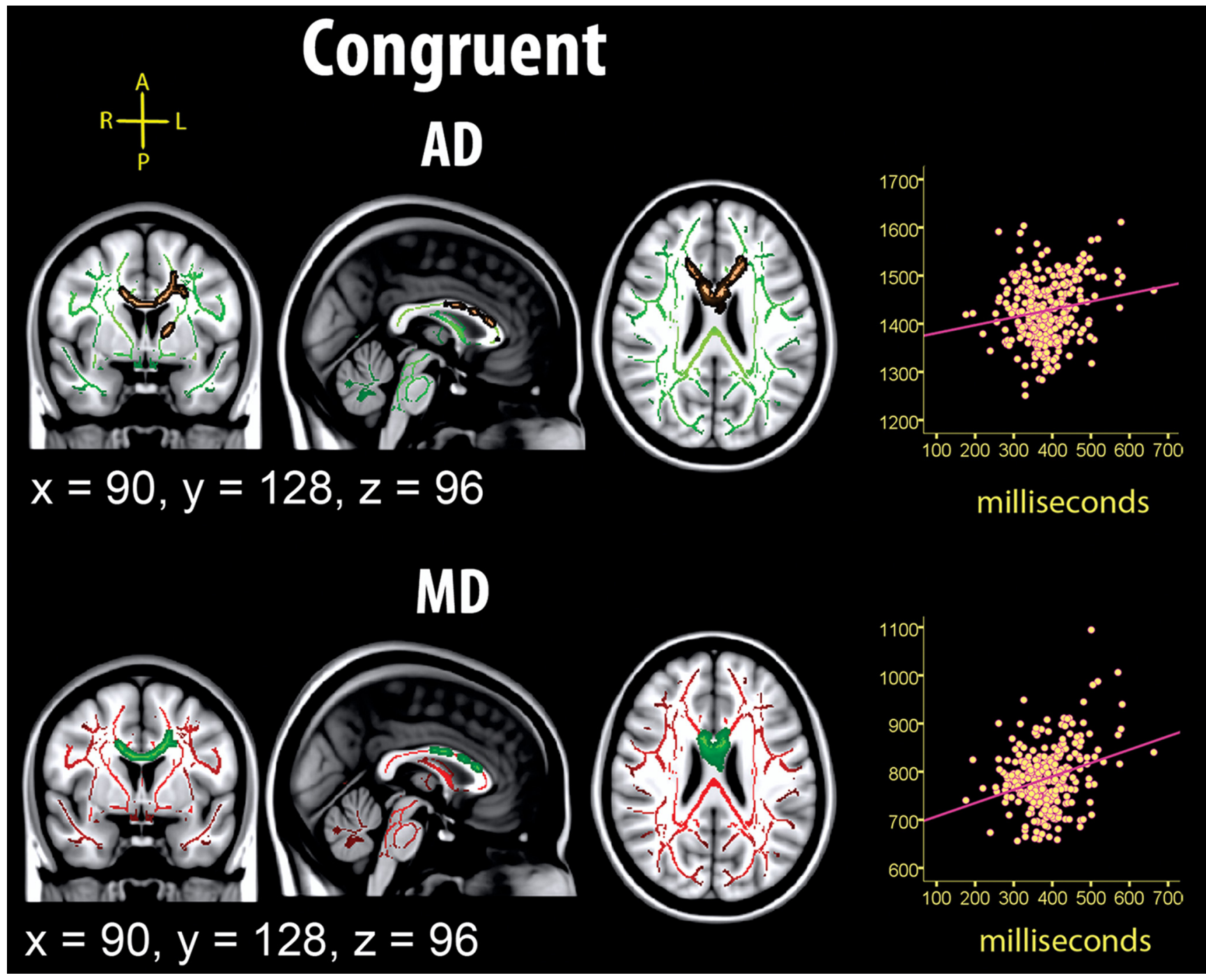

Figure 4. Relationship between $m R T$ in the congruent condition and diffusion characteristics. Left, Effects of mRT on diffusion characteristics, corrected for age and sex. The effects are corrected for multiple comparisons across space by threshold-free cluster enhancement at $p<0.05$. The results are smoothed to ease visualization of effects, and displayed on top of the WM skeleton (copper on green skeleton, $A D$; green on red skeleton, MD). No effects for FA or AD survived correction. Right, Scatterplots used to illustrate the raw data (in contrast to the other statistical analyses, age and sex are not used as covariates). The diffusion characteristics represent mean values across all voxels identified as statistically significant in the voxelwise skeleton analyses (fully corrected for multiple comparisons across space). 
Table 2. Size and spatial extension of effects

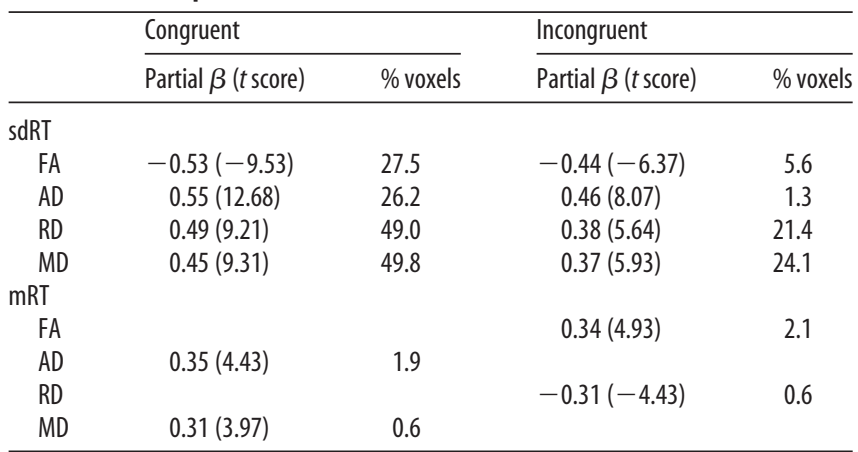

To illustrate the strength of the effects, multiple regression analyses were run. The partial $\beta$ s for sdRT are controlled for age, sex, and mRT; the partial $\beta$ s for mRT are controlled for age and sex. The partial $\beta$ statistics for the congruent condition are based on diffusion values from each voxel where a significant relationship of at least $p<0.001$ (uncorrected) between the RT measure and diffusion was found in the whole-skeleton analyses. For the incongruent condition, the effects were too weak to survive such a conservative threshold, so a threshold of $p<0.01$ was chosen to illustrate the effects. Based on the same reasoning, a threshold of 0.05 (corrected) was chosen for the mRT analyses. \% voxels denotes the percentage of the voxels in the skeleton that showed a significant relationship of at least $p=0.05$, fully corrected for multiple comparisons across space.

covering $17.8 \%$ (FA), $25.6 \%$ (AD), $43.7 \%$ (MD), and $41.8 \%$ (RD) of the skeleton voxels. The relationship between sdRT and WM diffusion characteristics increased through the age span, yielding very different slopes for the oldest versus the youngest part of the sample.

\section{Analyses of the extreme end of the sdRT distribution}

As seen from the scatterplots in Figure 3, some heteroscedasticity may exist in the IIV-WM diffusion models. To investigate the extreme end of the sdRT distribution further, we chose the $5 \%$ of the participants with the highest values $(>92 \mathrm{~ms}$ ) and compared these with the rest of the sample on age, sex, education, MMSE score, and full-scale IQ. Significant relationships between IIV and mean DTI values from voxels within effect sites (all voxels with $p<0.001$; Table 2) were seen in both groups (age, sex, and mRT as covariates), although they were substantially higher in the extreme group compared with the rest (FA: partial $\beta=-1.05$ vs -0.36 ; MD: $\operatorname{partial} \beta=0.91$ vs 0.24 ; $\mathrm{AD}$ : $\operatorname{partial} \beta=0.83$ vs 0.32 ; $\mathrm{RD}$ : partial $\beta=1.00$ vs 0.27 , all $p$ s $<0.05)$. The groups differed in age (extreme scorers 65.5 years vs normal scorers 47.7 years; Mann-Whitney $U$ test: $z=3.90, p<10^{-4}$ ), but not on any other of the tested variables (sex, education, MMSE score, and full-scale IQ). This confirms the results of the age-interaction analyses, where the relationships between IIV and DTI indices increase with age.

\section{Relationships between mRT and sdRT from the incongruent condition and DTI parameters}

Figure 7 shows the relationships between sdRT from the congruent condition and the different DTI parameters as color-coded overlays in the horizontal plane; scatterplots are shown in Figure 9. The results for mRT are shown in Figure 8.

Similar to the congruent condition, sdRT was negatively related to FA and positively to the other diffusion parameters, with no reverse relationships observed. Also in accordance with the results from the congruent condition, $\mathrm{RD}$ and $\mathrm{MD}$ showed the effects with the largest spatial extent. The spatial extent of the relationships were substantially smaller compared with the congruent condition, but $>20 \%$ of the skeleton voxels were still significantly related to sdRT for both RD and MD. To directly compare the distribution of effects along the posterior-toanterior axis across conditions, demeaned $t$ scores were plotted as a function of MNI $y$-coordinates; results are shown in Figure 5. As evident from this figure, the distribution of effect along the longitudinal axis did not differ much across conditions. For FA, a somewhat more anterior distribution of effects in the incongruent compared with the congruent condition was seen, corresponding to the results presented in Figure 7. For MD, this pattern was reversed. No formal statistical tests were performed to decide whether these minor differences were statistically significant.

For mRT, the results in the incongruent condition were less clear. In the TBSS analyses, a positive relationship between FA and $\mathrm{mRT}$ was found in parts of the right WM in relatively inferior areas spanning $2.1 \%$ of the skeleton voxels. However, when the correlation was recomputed without controlling for age and sex, a weak and not significant relationship was observed (Fig. 8). Thus, the positive relationship was only seen when strong statistical control for age and sex was imposed on the data. The same pattern of inconsistencies was observed for $\mathrm{RD}$, where a negative relationship was seen in TBSS. Like FA, the relationship vanished when age and sex were not used as covariates. These changes in direction of the relationships as a function of covariates render the $\mathrm{mRT}$ results in the incongruent condition hard to interpret.

\section{Discussion}

IIV was related to WM microstructure in large portions of the brain. Interestingly, the relationships were weaker and less consistent for speed of processing. Thus, measures of performance variability could be an at least as relevant a cognitive correlate of individual differences in WM integrity as information processing speed per se. Substantial research has focused on mapping the relationship between WM characteristics and speed of processing, and the factors that mediate this relationship. However, many cognitive tasks suitable for measuring processing speed can also be used to obtain measures of intraindividual performance variability. The present results suggest that performance variability represents an important supplement to understand the cognitive correlates of WM characteristics.

\section{WM characteristics and information processing speed}

Due to the spatial distribution of highly specialized brain areas (Gläscher et al., 2010) and that the main function of the myelin sheet is to isolate the axons, thereby facilitating conduction of action potentials over long distances, individual differences in WM structure are expected to affect cognitive performance, e.g., processing speed (O'Sullivan et al., 2001; Madden et al., 2004, 2009; Schulte et al., 2005; Deary et al., 2006; Grieve et al., 2007; Bucur et al., 2008; Correia et al., 2008; Turken et al., 2008; Kennedy and Raz, 2009; Vernooij et al., 2009). The present results confirm a relationship between $\mathrm{mRT}$ from the congruent condition and DTI parameters, in that significant effects were seen for $\mathrm{AD}$ and $\mathrm{MD}$ in the genu and middle sections (body) of the corpus callosum, and in the left anterior thalamic radiation and forceps minor for AD. Several studies report that various measures of information processing speed are related to microstructural characteristics of WM in the genu of corpus callosum (Bucur et al., 2008; Madden et al., 2009), in agreement with the present results from the congruent condition. However, the anatomical locations and which DTI parameter shows the strongest effects vary between studies, likely partly due to different cognitive tasks. This was evident in the present study, where the relationship between mRT and DTI was less clear in the incongruent condition. Positive relationships for FA and negative for RD were observed, but the direction changed when age and sex were not included in the model. These results demonstrate that variations in 
FA

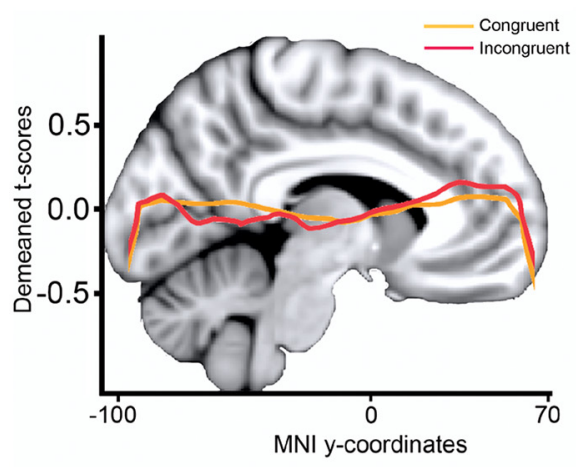

MD

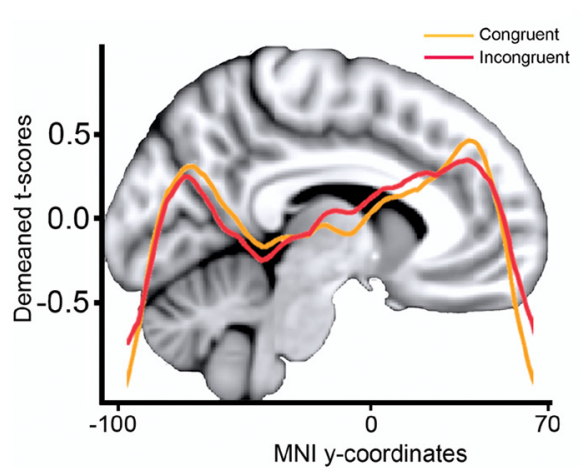

AD

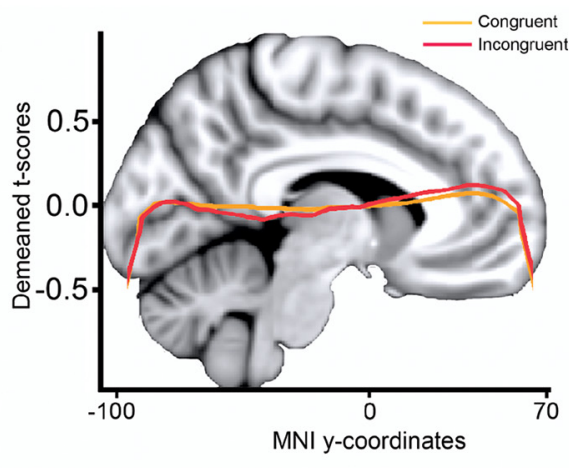

$\mathrm{RD}$

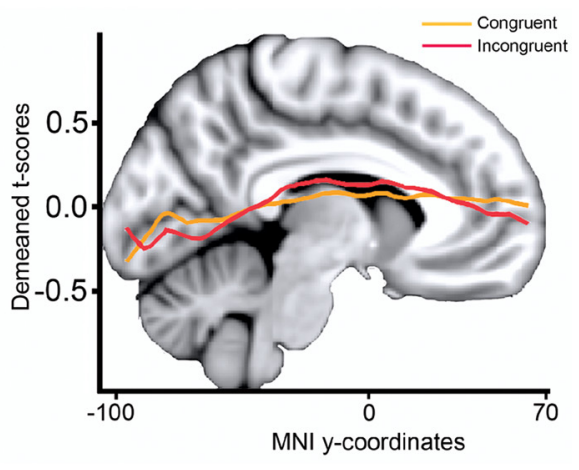

Figure 5. Posterior-to-anterior effects. Mean $t$ value in the skeleton at each integer MNI $y$-coordinate was calculated and fitted by locally weighted scatterplot smoothing. As can be seen from the locally weighted scatterplot smoothing curves, the effects are relatively similarly distributed in the posterior and anterior parts of the brain, and no clear difference in the posterior to anterior gradient can be seen between conditions.

the specific task used can substantially affect the results. More research is needed before we understand which areas of the WM can be singled out, which DTI parameters are the most relevant, and which factors impact the relationships with speed.

\section{WM characteristics and IIV}

Increased performance variability could be an equally likely consequence of lower WM integrity as slower processing speed, per se. WM changes, including volumetric decline, demyelination, hyperintensities, and FA, have been related to increased performance variability in previous studies (Britton et al., 1991; Anstey et al., 2007; Bunce et al., 2007; Walhovd and Fjell, 2007; Ullén et al., 2008; Moy et al., 2011). A recent DTI study yielded inconclusive results in that FA, but not other diffusion parameters, correlated with IIV (Moy et al., 2011). However, mean RT was not controlled for, and thus it is uncertain whether the effect could be explained by increased IIV as a function of increased RT. The present study establishes that relationships exist between all indices of microstructure across the WM and performance variability in a large sample of healthy individuals, that the relationship exists independently of processing speed, and that it increases with increasing age, but that the cognitive task used affects the results substantially.

The IIV-WM relationships support the theory that WM structural disconnectivity causes increased neural noise and performance variability (MacDonald et al., 2009). A recent study argued that increased variability in aging at the behavioral level may be caused by decreased variability in the BOLD response, possibly due to weakened functional connectivity (Garrett et al., 2011). The authors argued that greater BOLD variability represents a greater ability to transition between brain states. It can be speculated that lower structural connectivity yields lower functional connectivity, causing a less sophisticated and less complex neural system, lower brain functional variability (i.e., BOLD), and ultimately higher behavioral variability (sdRT).

An intriguing hypothesis is that the WM correlates of individual differences in cognitive functions change with age (Madden et al., 2004). In the present study, a clear age interaction was observed for all DTI parameters, where the relationship between IIV and WM microstructure increased with age. Weak relationships existed in the youngest half of the sample ( $<52$ years), in contrast to stronger relationships in the oldest part ( $\geq 52$ years). One interpretation of this finding is that increased IIV is a result of decreases in WM integrity, and that this relationship will be observed mainly in groups of participants where WM changes are observed. Thus, in young participants with high levels of WM integrity, weak relationships are observed, while in elderly participants, where individual differences in WM microstructural changes and sdRT may be larger, a relationship will emerge. In a previous study with an overlapping sample of participants, we observed an increased estimated rate of change in DTI parameters after 50-60 years of age (Westlye et al., 2010). This means that the average participant in the oldest part of the sample is more likely to experience WM microstructural changes at a rapid pace than the average participant in the youngest part. It is possible that such a mechanism is responsible for the increased IIV-WM relationships with higher age, but this needs to be tested directly with longitudinal data.

\section{Effects of cognitive demand}

Several studies indicate that IIV depends on factors such as task, practice (Allaire and Marsiske, 2005), and timescale (Li et al., 2004; Schmiedek et al., 2009). The inclusion of two conditions yielded the opportunity to test whether the results varied with degree of cognitive demands. The general tendencies were replicated, with robust relationships between IIV and WM diffusion across both conditions. However, the results were anatomically much more widespread in the congruent condition, affecting a higher percentage of the voxels. Thus, the cognitive task used to calculate IIV influenced the results, even though the main pattern of effects was similar. We speculated that higher attentional demands, including the more inhibitory processing required in the incongruent condition, could yield a relatively more anterior pattern of effects relative to the less demanding congruent condition. Although not formally statistically tested, the $t$ value gradient plots in Figure 5 indicate no clear posterior-anterior difference in effects across conditions. 

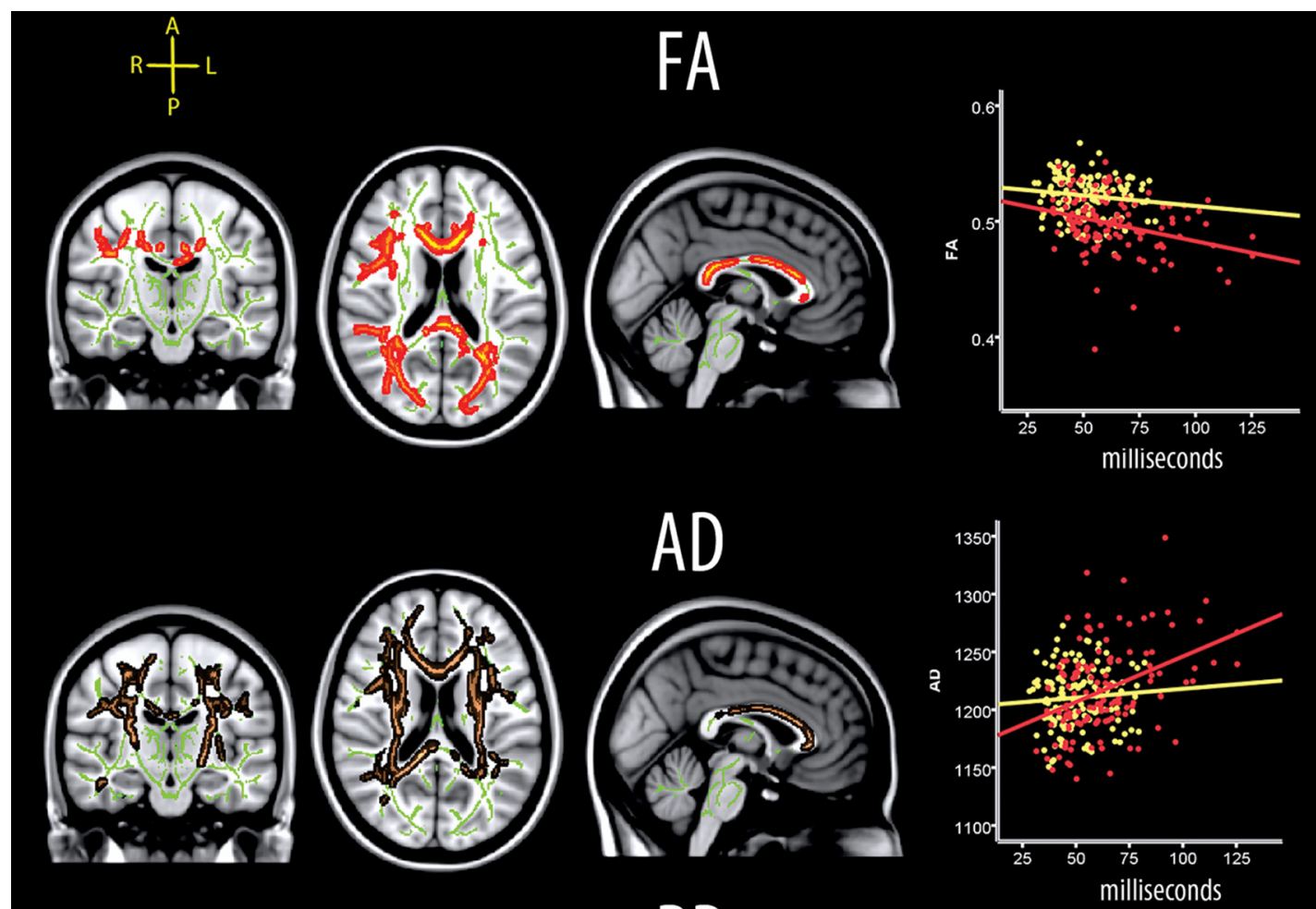

\section{$\mathrm{RD}$}
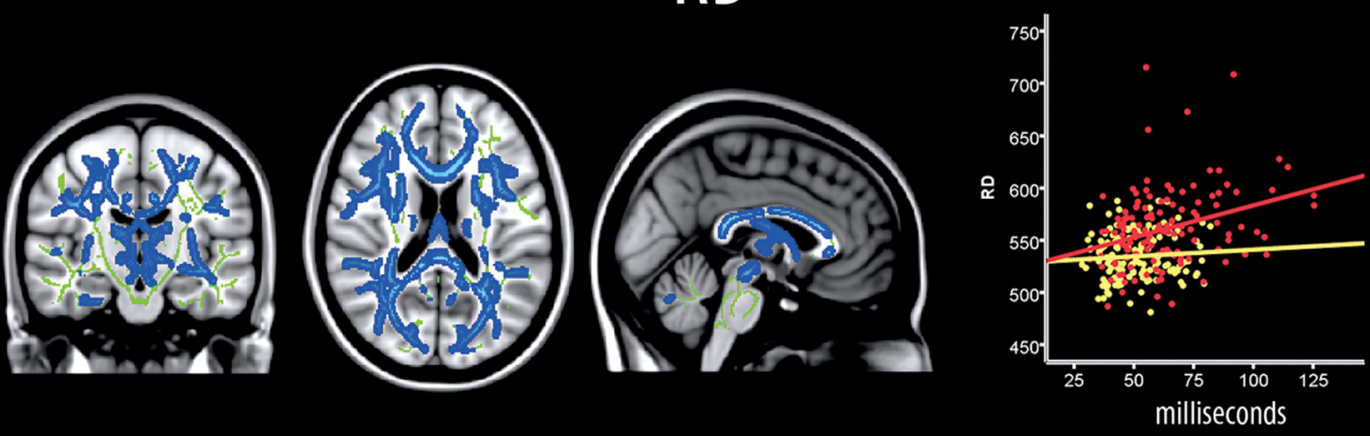

\section{MD}
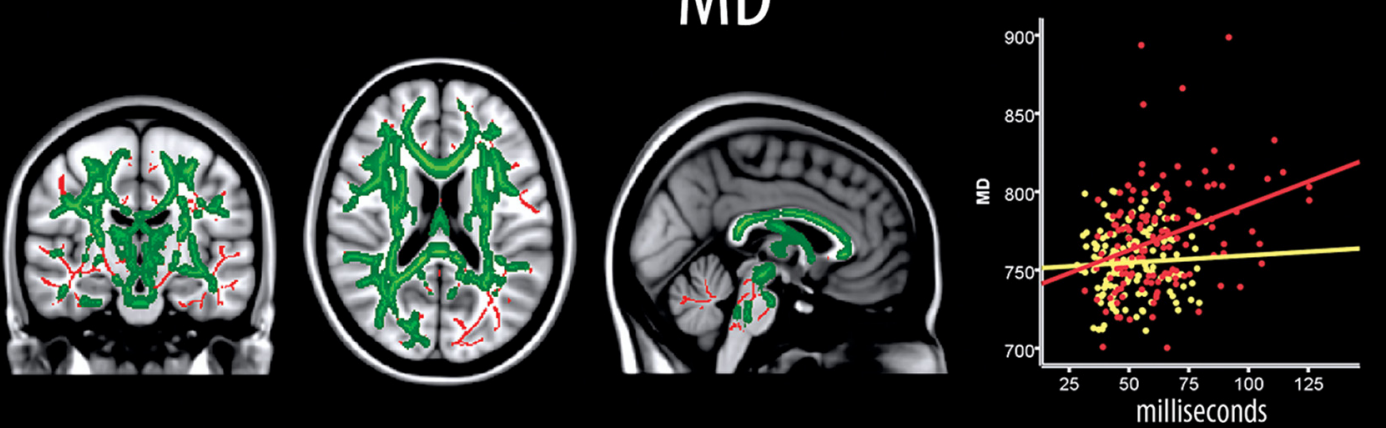

\section{Young ( $<52$ years)}

Elderly ( $\geq 52$ years)

Figure 6. Age interactions. Left, Voxels showing a significant age $\times$ sdRT interaction on diffusion characteristics are displayed (age, sex, $m R T$, and sdRT were used as covariates). The effects are corrected for multiple comparisons across space by threshold-free cluster enhancement at $p<0.05$. The results are smoothed to ease visualization of effects, and displayed on top of the WM skeleton (red on green skeleton, FA; copper on green skeleton, AD; blue on green skeleton, RD; green on red skeleton, MD). Right, Scatterplots illustrating the relationship between sdRT and diffusion characteristics across all voxels showing a significant relationship between sdRT and diffusion (Fig. 2). As can be seen, weak relationships exist in the youngest half of the sample (age, $<52$ years), while stronger relationships are seen in the oldest half (age, $\geq 52$ years). 


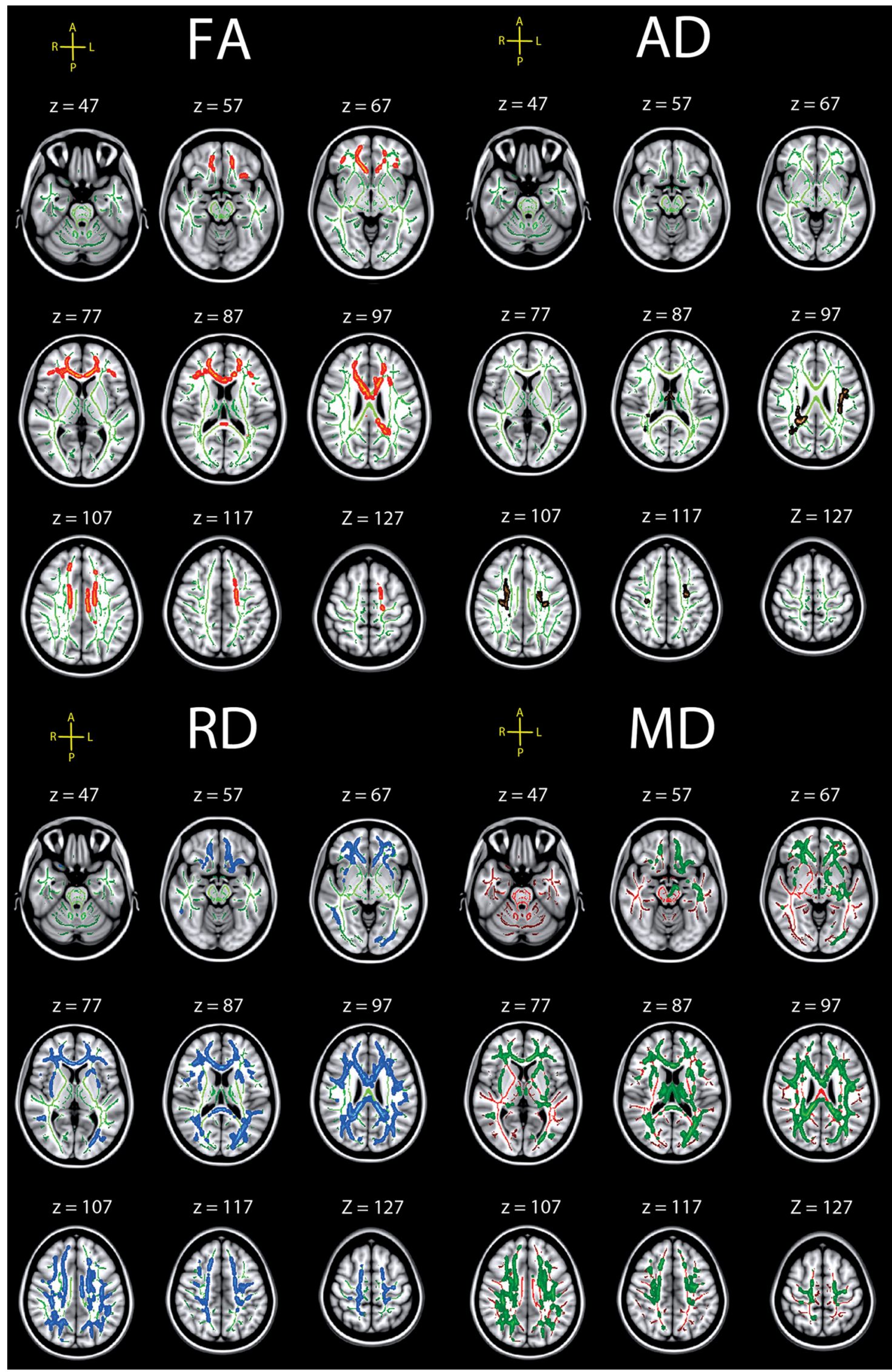

Figure 7. Effects of sdRT in the incongruent condition on diffusion characteristics. Effects of IIV from the incongruent condition on diffusion characteristics, corrected for age, sex, and mRT, corrected for multiple comparisons across space at $p<0.05$. The results are smoothed to ease visualization of effects, and displayed on top of the WM skeleton (red on green skeleton, FA; copper on green skeleton, $\mathrm{AD}$; blue on green skeleton, $\mathrm{RD}$; green on red skeleton, $\mathrm{MD}$ ). FA was negatively correlated with sdRT; the other diffusion measures were positively related. 


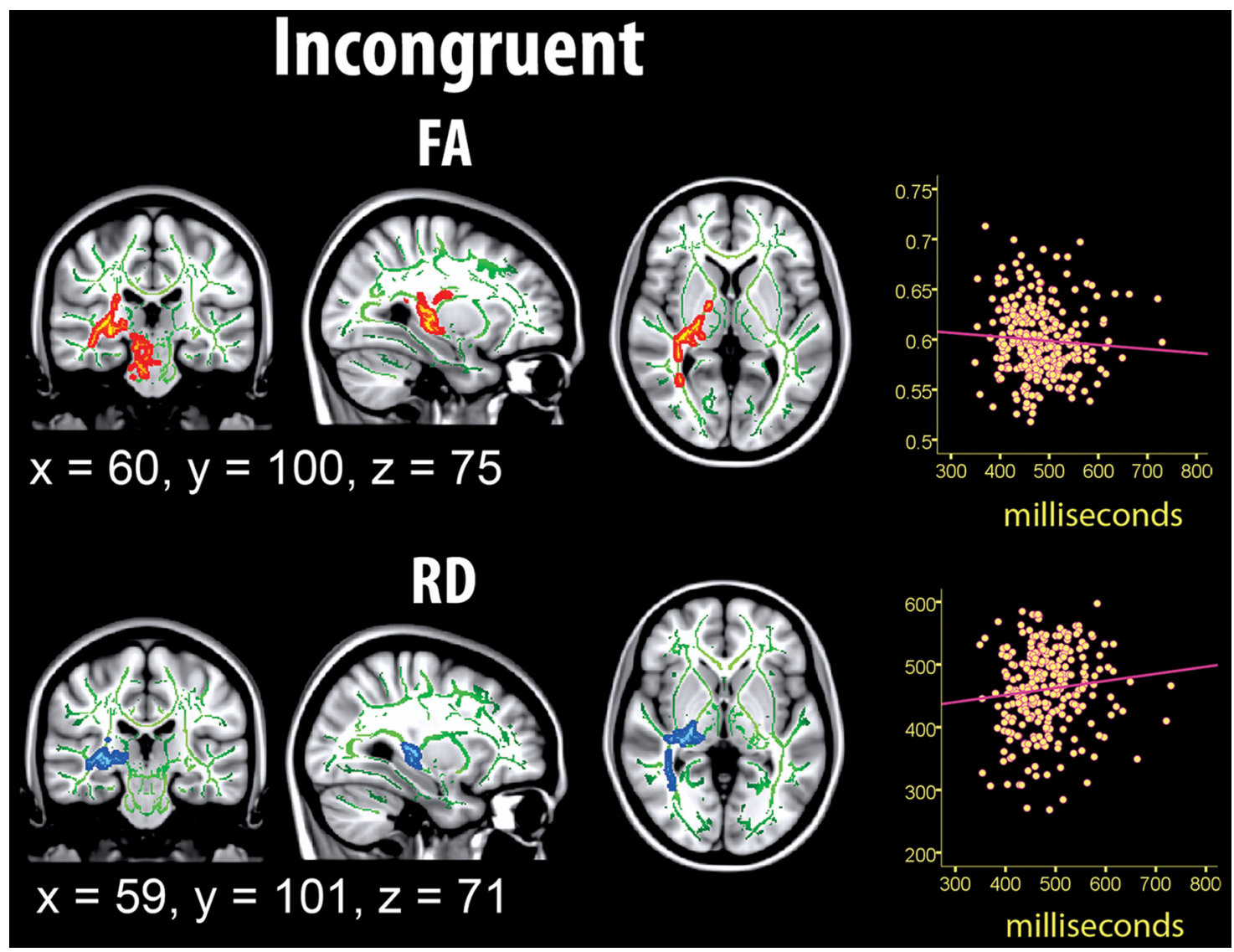

Figure 8. Relationship between $m R T$ in the incongruent condition and diffusion characteristics. Left, Effects of $m R T$ on diffusion characteristics, corrected for age and sex. The effects are corrected for multiple comparisons across space by threshold-free cluster enhancement at $p<0.05$. The results are smoothed to ease visualization of effects, and displayed on top of the WM skeleton (red on green skeleton, FA; blue on green skeleton, RD). No effects for AD and MD survived correction. Right, Scatterplots used to illustrate the raw data (in contrast to the other statistical analyses, age and sex were not used as covariates). The diffusion characteristics represent mean values across all voxels identified as statistically significant in the voxelwise skeleton analyses (fully corrected for multiple comparisons across space). Note that a positive relationship between FA and $\mathrm{mRT}$, and a negative relationship between RD and $\mathrm{mRT}$, is seen when the influence of age and sex are regressed out from $\mathrm{mRT}$.

\section{Incongruent}

MD

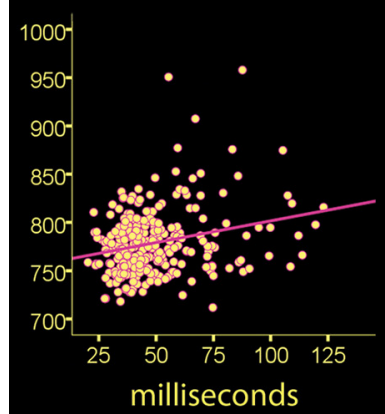

FA

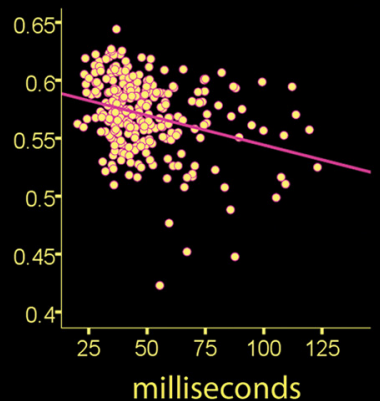

AD

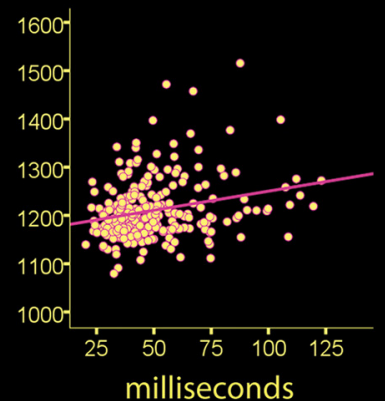

\section{RD}

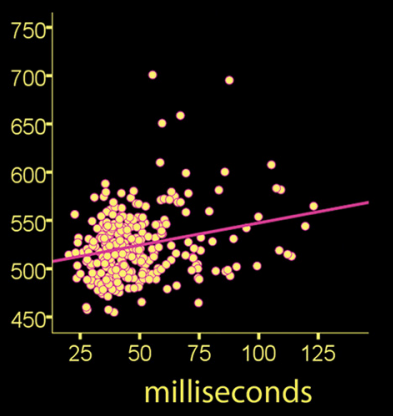

Figure 9. Scatterplots of sdRT in the incongruent condition and diffusion characteristics. Scatterplots illustrate the raw data, and thus age, sex, and mRT were not used as covariates, in contrast to the other statistical analyses. Four outliers were excluded from the analyses (sdRT $\pm 3 \mathrm{SD}$ from the mean). The diffusion characteristics represent mean values across all voxels identified as statistically significant in the voxelwise skeleton analyses (fully corrected for multiple comparisons across space).

Neurobiological underpinnings

DTI provides sensitive, but not very specific, indices of WM integrity. Four different DTI parameters were included, on the assumption that even though these are partly interdependent, they may yield complementary information. Axonal membranes are likely to be the primary determinant of FA, while myelin can modulate the degree of anisotropy in a given area of interest (Beaulieu, 2009). A recent histopathological study of temporal lobe epilepsy patients found axon membrane circumference to be most closely related to FA, with a high correlation observed also 
for axonal packing (Concha et al., 2010). Animal studies show that neurobiological events affect the diffusion parameters differently. In a classic series of studies, Song and colleagues showed that incomplete myelination increased $\mathrm{RD}$ but not $\mathrm{AD}$ (Song et al., 2002), while $\mathrm{AD}$ was more sensitive to axonal degeneration (Song et al., 2003). Still, myelin is not necessary to cause anisotropic diffusion, as evident in the brains of newborns. These contain little myelin but still show 54\% of adult FA (Provenzale et al., 2007). The nonmyelinated olfactory nerve of the garfish has higher anisotropy than the myelinated trigeminal and optical nerves, possibly related to the smaller axonal diameter in the former (Beaulieu, 2002). Also, lesions affect AD differently over time (Concha et al., 2006). We found most widespread DTI-IIV relationships for $\mathrm{MD}$ and $\mathrm{RD}$. While low $\mathrm{AD}$ in the mouse model described above (Song et al., 2002) was associated with axonal injury, AD correlated positively with IIV. Studies have shown AD increases in normal aging and Alzheimer's disease (Agosta et al., 2011; Madden et al., 2011). Positive relationships between AD and IIV explain why FA was less strongly related to IIV than MD or RD. Thus, the neurobiological characteristics more specifically related to FA may be less specific characteristics for IIV than degree of diffusivity along and across the principal axis of the diffusion tensor. The combination of effects on the different DTI parameters in the same voxels has also been used to draw specific inferences about the neurobiological foundations (Burzynska et al., 2010).

\section{Conclusion}

Relationships between performance variability and WM microstructure were found, indicating that cognitive variability may be at least as a relevant correlate of changes in WM microstructure as general processing speed per se. Further, the associations were found to increase with age, indicating that aging-related degenerative processes modulate the associations between IIV and WM microstructure. A limitation is that IIV may correlate with WM integrity due to shared variance with other brain correlates. This will be important to disentangle in future studies.

\section{References}

Agosta F, Pievani M, Sala S, Geroldi C, Galluzzi S, Frisoni GB, Filippi M (2011) White matter damage in Alzheimer disease and its relationship to gray matter atrophy. Radiology 258:853-863.

Allaire JC, Marsiske M (2005) Intraindividual variability may not always indicate vulnerability in elders' cognitive performance. Psychol Aging 20:390-401.

Andersson JL, Jenkinson M, Smith S (2007a) Non-linear optimisation. In: FMRIB technical report TR07JA1. wwwfmriboxacuk/analysis/techrep.

Andersson JL, Jenkinson M, Smith S (2007b) Non-linear registration, aka spatial normalization. In: FMRIB technical report TR07JA2. wwwfmriboxacuk/analysis/techrep.

Anstey KJ, Mack HA, Christensen H, Li SC, Reglade-Meslin C, Maller J, Kumar R, Dear K, Easteal S, Sachdev P (2007) Corpus callosum size, reaction time speed and variability in mild cognitive disorders and in a normative sample. Neuropsychologia 45:1911-1920.

Bäckman L, Nyberg L, Lindenberger U, Li SC, Farde L (2006) The correlative triad among aging, dopamine, and cognition: current status and future prospects. Neurosci Biobehav Rev 30:791-807.

Beaulieu C (2002) The basis of anisotropic water diffusion in the nervous system: a technical review. NMR Biomed 15:435-455.

Beaulieu C (2009) The biological basis of diffusion anisotropy. In: Diffusion MRI (Johansen-Berg H, Behrens T, eds), pp 105-126. London: Academic.

Beck AT, Steer R (1987) Beck depression inventory scoring manual. New York: Psychological Corporation.

Botvinick MM, Braver TS, Barch DM, Carter CS, Cohen JD (2001) Conflict monitoring and cognitive control. Psychol Rev 108:624-652.

Bravo G, Hébert R (1997) Age-and education-specific reference values for the Mini-Mental and modified Mini-Mental State Examinations derived from a non-demented elderly population. Int J Geriatr Psychiatry 12:1008-1018.

Britton TC, Meyer BU, Benecke R (1991) Variability of cortically evoked motor responses in multiple sclerosis. Electroencephalogr Clin Neurophysiol 81:186-194.

Bucur B, Madden DJ, Spaniol J, Provenzale JM, Cabeza R, White LE, Huettel SA (2008) Age-related slowing of memory retrieval: contributions of perceptual speed and cerebral white matter integrity. Neurobiol Aging 29:1070-1079.

Bunce D, Anstey KJ, Christensen H, Dear K, Wen W, Sachdev P (2007) White matter hyperintensities and within-person variability in communitydwelling adults aged 60-64 years. Neuropsychologia 45:2009-2015.

Burzynska AZ, Preuschhof C, Bäckman L, Nyberg L, Li SC, Lindenberger U, Heekeren HR (2010) Age-related differences in white matter microstructure: region-specific patterns of diffusivity. Neuroimage 49:2104-2112.

Concha L, Gross DW, Wheatley BM, Beaulieu C (2006) Diffusion tensor imaging of time-dependent axonal and myelin degradation after corpus callosotomy in epilepsy patients. Neuroimage 32:1090-1099.

Concha L, Livy DJ, Beaulieu C, Wheatley BM, Gross DW (2010) In vivo diffusion tensor imaging and histopathology of the fimbria-fornix in temporal lobe epilepsy. J Neurosci 30:996-1002.

Correia S, Lee SY, Voorn T, Tate DF, Paul RH, Zhang S, Salloway SP, Malloy PF, Laidlaw DH (2008) Quantitative tractography metrics of white matter integrity in diffusion-tensor MRI. Neuroimage 42:568 -581.

Deary IJ, Bastin ME, Pattie A, Clayden JD, Whalley LJ, Starr JM, Wardlaw JM (2006) White matter integrity and cognition in childhood and old age. Neurology 66:505-512.

Debener S, Ullsperger M, Siegel M, Fiehler K, von Cramon DY, Engel AK (2005) Trial-by-trial coupling of concurrent electroencephalogram and functional magnetic resonance imaging identifies the dynamics of performance monitoring. J Neurosci 25:11730-11737.

Eriksen BA, Eriksen CW (1974) Effects of noise letters upon the identification of a target letter in a nonsearch task. Percept Psychophys 16:143-149.

Fjell AM, Westlye LT, Greve DN, Fischl B, Benner T, van der Kouwe AJ, Salat D, Bjornerud A, Due-Tønnessen P, Walhovd KB (2008) The relationship between diffusion tensor imaging and volumetry as measures of white matter properties. Neuroimage 42:1654-1668.

Folstein MF, Folstein SE, McHugh PR (1975) "Mini-mental state" : a practical method for grading the cognitive state of patients for the clinician. J Psychiatr Res 12:189-198.

Garrett DD, Kovacevic N, McIntosh AR, Grady CL (2011) The importance of being variable. J Neurosci 31:4496-4503.

Gläscher J, Rudrauf D, Colom R, Paul LK, Tranel D, Damasio H, Adolphs R (2010) Distributed neural system for general intelligence revealed by lesion mapping. Proc Natl Acad Sci U S A 107:4705-4709.

Grieve SM, Williams LM, Paul RH, Clark CR, Gordon E (2007) Cognitive aging, executive function, and fractional anisotropy: a diffusion tensor MR imaging study. AJNR Am J Neuroradiol 28:226-235.

Hofer SM, Sliwinski MJ (2001) Understanding ageing: an evaluation of research designs for assessing the interdependence of ageing-related changes. Gerontology 47:341-352.

Hultsch DF, MacDonald SW (2004) Intraindividual variability in performance as a theoretical window onto cognitive aging. In: New frontiers in cognitive aging (Dixon R, Backman L, Nilsson LG, eds), pp 65-88. Oxford: Oxford UP.

Jenkinson M, Smith S (2001) A global optimisation method for robust affine registration of brain images. Med Image Anal 5:143-156.

Kelly AM, Uddin LQ, Biswal BB, Castellanos FX, Milham MP (2008) Competition between functional brain networks mediates behavioral variability. Neuroimage 39:527-537.

Kennedy KM, Raz N (2009) Aging white matter and cognition: differential effects of regional variations in diffusion properties on memory, executive functions, and speed. Neuropsychologia 47:916-927.

Li SC, Huxhold O, Schmiedek F (2004) Aging and attenuated processing robustness. Evidence from cognitive and sensorimotor functioning. Gerontology 50:28-34.

Lövdén M, Li SC, Shing YL, Lindenberger U (2007) Within-person trial-totrial variability precedes and predicts cognitive decline in old and very old age: longitudinal data from the Berlin Aging Study. Neuropsychologia 45:2827-2838.

MacDonald SW, Nyberg L, Bäckman L (2006) Intra-individual variability 
in behavior: links to brain structure, neurotransmission and neuronal activity. Trends Neurosci 29:474-480.

MacDonald SW, Li SC, Bäckman L (2009) Neural underpinnings of withinperson variability in cognitive functioning. Psychol Aging 24:792-808.

Madden DJ, Whiting WL, Huettel SA, White LE, MacFall JR, Provenzale JM (2004) Diffusion tensor imaging of adult age differences in cerebral white matter: relation to response time. Neuroimage 21:1174-1181.

Madden DJ, Spaniol J, Costello MC, Bucur B, White LE, Cabeza R, Davis SW, Dennis NA, Provenzale JM, Huettel SA (2009) Cerebral white matter integrity mediates adult age differences in cognitive performance. J Cogn Neurosci 21:289-302.

Madden DJ, Bennett IJ, Burzynska A, Potter GG, Chen NK, Song AW (2011) Diffusion tensor imaging of cerebral white matter integrity in cognitive aging. Biochim Biophys Acta. Advance online publication. doi:10.1016/j.bbadis.2011.08.003.

Moy G, Millet P, Haller S, Baudois S, de Bilbao F, Weber K, Lövblad K, Lazeyras F, Giannakopoulos P, Delaloye C (2011) Magnetic resonance imaging determinants of intraindividual variability in the elderly: combined analysis of grey and white matter. Neuroscience 186:88-93.

Nichols TE, Holmes AP (2002) Nonparametric permutation tests for functional neuroimaging: a primer with examples. Hum Brain Mapp 15:1-25.

O'Sullivan M, Jones DK, Summers PE, Morris RG, Williams SC, Markus HS (2001) Evidence for cortical "disconnection" as a mechanism of agerelated cognitive decline. Neurology 57:632-638.

Provenzale JM, Liang L, DeLong D, White LE (2007) Diffusion tensor imaging assessment of brain white matter maturation during the first postnatal year. AJR Am J Roentgenol 189:476-486.

Reese TG, Heid O, Weisskoff RM, Wedeen VJ (2003) Reduction of eddycurrent-induced distortion in diffusion MRI using a twice-refocused spin echo. Magn Reson Med 49:177-182.

Rueckert D, Sonoda LI, Hayes C, Hill DL, Leach MO, Hawkes DJ (1999) Nonrigid registration using free-form deformations: application to breast MR images. IEEE Trans Med Imaging 18:712-721.

Schmiedek F, Lövdén M, Lindenberger U (2009) On the relation of mean reaction time and intraindividual reaction time variability. Psychol Aging 24:841-857.

Schulte T, Sullivan EV, Müller-Oehring EM, Adalsteinsson E, Pfefferbaum A (2005) Corpus callosal microstructural integrity influences interhemispheric processing: a diffusion tensor imaging study. Cereb Cortex 15:1384-1392.

Smith SM (2002) Fast robust automated brain extraction. Hum Brain Mapp 17:143-155.

Smith SM, Nichols TE (2009) Threshold-free cluster enhancement: addressing problems of smoothing, threshold dependence and localisation in cluster inference. Neuroimage 44:83-98.

Smith SM, Jenkinson M, Woolrich MW, Beckmann CF, Behrens TE, Johansen-Berg H, Bannister PR, De Luca M, Drobnjak I, Flitney DE, Niazy RK, Saunders J, Vickers J, Zhang Y, De Stefano N, Brady JM, Mat- thews PM (2004) Advances in functional and structural MR image analysis and implementation as FSL. Neuroimage 23 [Suppl 1]:S208-S219.

Smith SM, Jenkinson M, Johansen-Berg H, Rueckert D, Nichols TE, Mackay CE, Watkins KE, Ciccarelli O, Cader MZ, Matthews PM, Behrens TE (2006) Tract-based spatial statistics: voxelwise analysis of multi-subject diffusion data. Neuroimage 31:1487-1505.

Smith SM, Johansen-Berg H, Jenkinson M, Rueckert D, Nichols TE, Miller KL, Robson MD, Jones DK, Klein JC, Bartsch AJ, Behrens TE (2007) Acquisition and voxelwise analysis of multi-subject diffusion data with tract-based spatial statistics. Nat Protoc 2:499-503.

Song SK, Sun SW, Ramsbottom MJ, Chang C, Russell J, Cross AH (2002) Dysmyelination revealed through MRI as increased radial (but unchanged axial) diffusion of water. Neuroimage 17:1429-1436.

Song SK, Sun SW, Ju WK, Lin SJ, Cross AH, Neufeld AH (2003) Diffusion tensor imaging detects and differentiates axon and myelin degeneration in mouse optic nerve after retinal ischemia. Neuroimage 20:1714-1722.

Stuss DT, Murphy KJ, Binns MA, Alexander MP (2003) Staying on the job: the frontal lobes control individual performance variability. Brain 126:2363-2380.

Turken A, Whitfield-Gabrieli S, Bammer R, Baldo JV, Dronkers NF, Gabrieli JD (2008) Cognitive processing speed and the structure of white matter pathways: convergent evidence from normal variation and lesion studies. Neuroimage 42:1032-1044.

Ullén F, Forsman L, Blom O, Karabanov A, Madison G (2008) Intelligence and variability in a simple timing task share neural substrates in the prefrontal white matter. J Neurosci 28:4238-4243.

Vernooij MW, Ikram MA, Vrooman HA, Wielopolski PA, Krestin GP, Hofman A, Niessen WJ, Van der Lugt A, Breteler MM (2009) White matter microstructural integrity and cognitive function in a general elderly population. Arch Gen Psychiatry 66:545-553.

Walhovd KB, Fjell AM (2007) White matter volume predicts reaction time instability. Neuropsychologia 45:2277-2284.

Wechsler D (1999) Wechsler Abbreviated Scale of Intelligence. San Antonio, TX: Psychological Corporation.

West R, Murphy KJ, Armilio ML, Craik FI, Stuss DT (2002) Lapses of intention and performance variability reveal age-related increases in fluctuations of executive control. Brain Cogn 49:402-419.

Westlye LT, Walhovd KB, Bjørnerud A, Due-Tønnessen P, Fjell AM (2009) Error-related negativity is mediated by fractional anisotropy in the posterior cingulate gyrus: a study combining diffusion tensor imaging and electrophysiology in healthy adults. Cereb Cortex 19:293-304.

Westlye LT, Walhovd KB, Dale AM, Bjørnerud A, Due-Tønnessen P, Engvig A, Grydeland H, Tamnes CK, Ostby Y, Fjell AM (2010) Life-span changes of the human brain white matter: diffusion tensor imaging (DTI) and volumetry. Cereb Cortex 20:2055-2068.

Woolrich MW, Jbabdi S, Patenaude B, Chappell M, Makni S, Behrens T, Beckmann C, Jenkinson M, Smith SM (2009) Bayesian analysis of neuroimaging data in FSL. Neuroimage 45:S173-S186. 